\title{
Nonadiabatic effects in two-level systems: A classical analysis
}

\author{
Marc Boiron, Maurice Lombardi, and Laurent Wiesenfeld \\ Laboratoire de Spectrométrie Physique, Université Joseph-Fourier-Grenoble, Boîte Postale 87, \\ F-38402 Saint-Martin-d'Hères Cédex, France
}

(Received 13 October 1993)

\begin{abstract}
Our aim in this paper is to study classical dynamics in two-level molecular systems. We first derive, through the Wigner phase-space transform, a classical limit that reduces unfortunately to the adiabatic approximation. This leads us to develop a one-center quantal approximation whose variables can be interpreted in a fully classical Hamiltonian scheme. As a prominent feature, this Hamiltonian couples polarization and spatial motion. We apply it successfully to the RosenthalStückelberg oscillations. We analyze one-dimensional diffusion similar to a molecular reaction; it appears to exhibit chaotic behavior.
\end{abstract}

PACS number(s): 34.10.+x, 03.65.Sq, 05.45.+b, 34.50.-s

\section{INTRODUCTION}

It has been well known for a long time that nonadiabatic effects may occur when two diabatic surfaces of potential cross each other. Various transition probabilities between the two surfaces may be calculated using Landau-Zener type of approaches, with much sophistication included [1-3]. The degrees of freedom of the system are divided into two sets, which eventually will be treated in a different way. Slow degrees of freedom, associated often with heavy particles, will somehow be considered as classical or semiclassical, whereas the fast motion of the light particles are considered as fully quantal. The very intuitive notion of a trajectory in a chemical reaction originates from this natural distinction.

In atomic and molecular physics, it is thus customary to associate the fast degree of freedom with the Bohr frequency associated with the two electronic energy levels, whereas the low frequencies result from the quantification of the nuclear motion in the spatially extended potential sheets (see Fig. 1). On that image is built the usual Born-Oppenheimer or adiabatic approximation, as well as the opposite diabatic approximation. It is also well known that those approximations break down in several instances : simple Landau-Zener crossings [1], conical intersections [5], or Jahn-Teller effects [6], electronic coherences between the two levels or Rosenthal-Stückelberg oscillations [7]. Also, classical dynamics on two surfaces, allowing for hopping in a Landau-Zener scheme has been addressed to, in order to see the appearance of classical chaos [8]. Because of the complexity of the quantum evolution equations, especially in several spatial dimensions, there has been several attempts to reduce the problem to a "hemi-quantal" one $[9,10]$. The fast sector is treated as fully quantum as possible, whereas the slow sector is treated classically. Resulting equations couple ordinary and partial differential equations in a self-consistent way. For the problem that we are dealing with, this line of thought is all the more interesting as it may shed light on the role of non-adiabatic transitions in the appearance of vibronic chaos (the $\mathrm{NO}_{2}$ molecule case [11]) or diffusive chaos on two potential sheets (as explained below). In this paper we shall consequently make the assumption that the electronic degrees of freedom should be treated in a different way than the nuclear degrees of freedom. If we keep the idea of discrete levels for the electrons, without further approximations, it will be shown in a first part that the limit at small $\hbar$ is simply the adiabatic limit, which unfortunately is of no interest for us. Then, in a second part, we make the main approximation, that the nuclear wave packet remains centered around one and the same point that evolutes in time for any value of the electronic energy. Then, a Bloch sphere model is straightforward to derive. We shall show that even this model, completely classical in its expression, is capable to reproduce the Rosenthal-Stückelberg oscillations. As a by-product, it will be shown that the coupling of the two degrees of freedom is enough to generate classical chaos, in the diffusive regime for example. Some

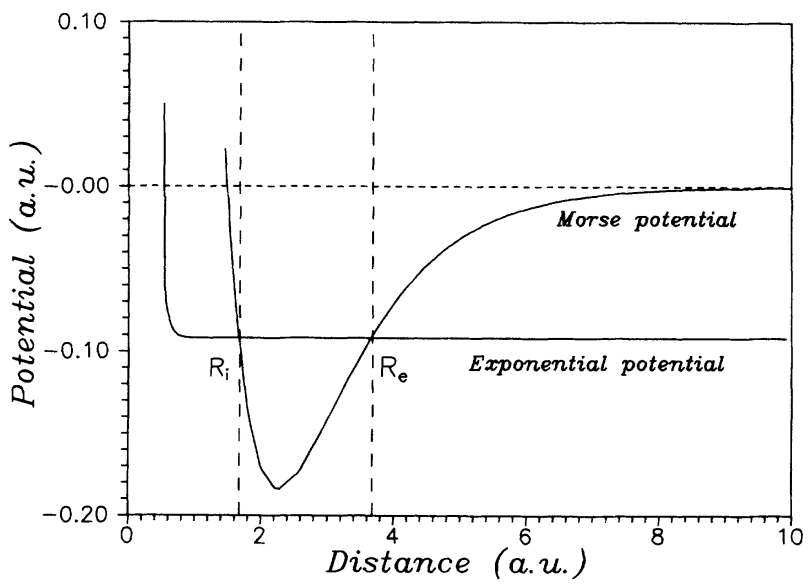

FIG. 1. An example of a relevant two-sheet potential in reactive collision; only a one-dimensional cut is shown. Potentials are shown in diabatic base, so that the two potential lines exactly cross each other at $R=R_{i}, R_{e}$. The coupling potential is not represented. 
similar approaches exist in the literature [12,13], putting the emphasis on different parts of the formalism.

It is clear that the approach presented here belongs to the family of hemi-quantal problems. Dealing with a very special quantum sector, namely spin, we get rid of any partial differential equation, and our classical model is fully Hamiltonian. As will be explained in detail, our approximation lies in between the spin $1 / 2$ particle moving in an adiabatic way in a space-varying magnetic field and the fully diabatic Landau-Zener treatment.

The adiabatic case has been known in the literature for some time in different contexts (in molecular physics, see for example [14]). We feel that for sake of comparison, it is most useful to compare here in detail the adiabatic and hemi-quantal approximations and especially to show where they depart one from another.

\section{CLASSICAL AND SEMICLASSICAL HAMILTONIANS}

The Hamiltonian to be considered is

$$
\mathcal{H}=-\frac{\hbar^{2}}{2 M} \frac{\partial^{2}}{\partial \mathbf{R}^{2}}-\frac{\hbar^{2}}{2 m} \frac{\partial^{2}}{\partial \mathbf{r}^{2}}+V(\mathbf{R}, \mathbf{r})
$$

where $\mathbf{R}$ and $M$ denote nuclear coordinates and masses, collectively, and $\mathbf{r}$ and $m$, the electronic coordinates and mass. We make the very general assumption of two different scales of quantized energy, associated with electrons on one hand and with translational energy of nuclei on the other hand. The nuclear scale of quantized energy is given by $\hbar \omega_{N}$ while the electronic scale is given by $\hbar \omega_{e}$. In order to make the various semiclassical limits that we shall explain in the following, let us precise here the domains of relevance. We shall consider that in general the de Broglie wavelength $\lambda_{N}=h / p_{N}=h / m_{N} v_{N}$ of the nuclear wave is much shorter than its electronic counterpart $\lambda_{e}=h / p_{e}=h / m_{e} v_{e}$, simply because of the mass ratio $\chi=m_{e} / m_{N} \ll 1$. The length scale associated with the variation of the electronic potentials is supposed to be always much larger than the $\lambda_{N}$ wavelength. A semiclassical formalism in the nuclear sector seems, therefore, fully justified. The difficulties with semiclassical images arise when a nuclear wave function is coupled to several electronic levels. Several types of formalisms have been proposed to deal with the two-level systems, either by making use of the adiabatic approximation, or by making hybrid semiclassical limits.

\section{A. Spinorial formalism: Adiabatic limit}

In a first part, we shall try to push the quantum formalism as far as possible while eventually keeping only the leading terms in $\hbar$. In order to separate the nuclear and electronic degrees of freedom, let us as usual develop the total wave function $\Psi$ in a so-called diabatic base (no approximation here):

$$
\Psi(\mathbf{R}, \mathbf{r})=\sum_{i} c_{i}(\mathbf{R}) \varphi_{i}\left(\mathbf{r}, \mathbf{R}_{0}\right)
$$

where the electronic wave functions $\varphi_{i}$ are taken at a fixed, arbitrary configuration of the nuclei, given by $\mathbf{R}_{0}$. Supposing for sake of definiteness time-reversal invariance and only two electronic states, the quantum equation of motion for the $c_{i}(\mathbf{R})$ are, in matrix form,

$$
\begin{aligned}
i \hbar \frac{d}{d t}\left|\begin{array}{l}
c_{1}(\mathbf{R}, t) \\
c_{2}(\mathbf{R}, t)
\end{array}\right\rangle= & {\left[\left(\frac{\mathbf{P}^{2}}{2 M}+V_{0}(\mathbf{R})\right)\left(\begin{array}{ll}
1 & 0 \\
0 & 1
\end{array}\right)\right.} \\
& +V_{z}(\mathbf{R}) \frac{1}{2}\left(\begin{array}{cc}
1 & 0 \\
0 & -1
\end{array}\right) \\
& \left.+V_{x}(\mathbf{R}) \frac{1}{2}\left(\begin{array}{ll}
0 & 1 \\
1 & 0
\end{array}\right)\right]\left|\begin{array}{l}
c_{1}(\mathbf{R}, t) \\
c_{2}(\mathbf{R}, t)
\end{array}\right\rangle .
\end{aligned}
$$

$V_{a}(\mathbf{R}), a=x, y, z$, is a pseudomagnetic field (units of energy), that represents the potential coupling between the electronic states 1 and 2 , represented by the spinor $\left|c_{1} c_{2}\right\rangle$. The vector $\vec{V}$ lives in a three-dimensional (3D) space, of course not to be confused with ordinary space in which vectors $\mathbf{R}$ live. Equation (3) may be seen as the quantum evolution equation of a spin $1 / 2$ particle, subjected to a scalar potential $V_{0}(\mathbf{R})$ and to an inhomogeneous pseudomagnetic field $\vec{V}(\mathbf{R})$.

Let us now summarize the main steps of the derivation of the classical limit of the evolution Eq. (3), through Wigner transform theory [15]. It leads to recast (3) in the density matrix formalism. The more adapted representation for the spinorial part of the density matrix is the base composed of the unity and dimentionless spin $1 / 2$ matrices: $\left\{\sigma_{0}=1, S_{a}=1 / 2 \sigma_{a}, a=x, y, z\right\}$ where the $\sigma_{a}$ are the Pauli matrices. The components of the density matrix $\varrho$ in this base are

$$
\begin{aligned}
& \rho_{0}\left(\mathbf{R}, \mathbf{R}^{\prime}, t\right)=\operatorname{Tr}_{2}\left[\varrho \sigma_{0}\right] \\
& \rho_{a}\left(\mathbf{R}, \mathbf{R}^{\prime}, t\right)=\operatorname{Tr}_{2}\left[\varrho S_{a}\right],
\end{aligned}
$$

where $\operatorname{Tr}_{2}$ is the trace over the $2 \times 2$ matrices, and the Hamiltonian reads

$$
\mathcal{H}=\left(\frac{\mathbf{P}^{2}}{2 M}+V_{0}(\mathbf{R})\right) \sigma_{0}+\sum_{a} V_{a}(\mathbf{R}) S_{a} .
$$

Substituting into (3), we get an equation for each component

$$
\begin{aligned}
& i \hbar \frac{\partial \rho_{0}}{\partial t}=\left[H_{0}, \rho_{0}\right]+\sum_{b}\left[V_{b}, \rho_{b}\right], \\
& i \hbar \frac{\partial \rho_{a}}{\partial t}=\left[H_{0}, \rho_{a}\right]+\frac{1}{4}\left[V_{a}, \rho_{0}\right]+i \sum_{b, c} \epsilon_{a b c}\left[V_{b}, \rho_{c}\right]_{+},
\end{aligned}
$$

where $[,]_{+}$denotes an anticommutator, $\epsilon_{a b c}$ is the completely antisymmetric tensor and $H_{0}$ is the $\sigma_{0}$ component of $\mathcal{H}$. The Wigner transform of (6) and (7) changes the components of $\mathcal{H}$ into their classical counterparts (function of $\mathbf{R}$ and/or $\mathbf{P}$ ) and the $\rho_{0, a}$ matrices become distribution functions $\gamma_{0, a}(\mathbf{R}, \mathbf{P}, t)$. Keeping the leading terms in $\hbar$, we obtain

$$
\frac{\partial \gamma_{0}}{\partial t}=\left\{H_{0}, \gamma_{0}\right\}+\sum_{b}\left\{V_{b}, \gamma_{b}\right\}
$$




$$
\frac{\partial \gamma_{a}}{\partial t}=\left\{H_{0}, \gamma_{a}\right\}+\frac{1}{4}\left\{V_{a}, \gamma_{0}\right\}+\frac{1}{\hbar} \sum_{b, c} \epsilon_{a b c} V_{b} \gamma_{c}
$$

where $\{$,$\} is the Poisson bracket taken over the variables$ $\mathbf{R}, \mathbf{P}$.

Such a process, applied to a scalar Hamiltonian, leads to the Liouville equations; hence, it is a way of taking the classical limit of the Schrödinger equation. Let us discuss the spinorial case. The limit at $\hbar \rightarrow 0$ of Eq. (8) is easy, as all leading terms are of the same order in $\hbar$. Equation (9) is more delicate to understand. Let us proceed to show that it reduces to the adiabatic approximation. We rewrite the last term as $\frac{1}{\hbar} \vec{V} \times \vec{\gamma}$. For the projection of $\vec{\gamma}$ on the perpendicular plane to $\vec{V}$, the dominant term in Eq. (9) is the cross product in $1 / \hbar$, meaning a precession of $\vec{\gamma}$ around $\vec{V}$ faster than any other motion in the dynamics. Otherwise stated, all spin components orthogonal to $\vec{V}$ are averaged to zero, for any practical purposes. The only meaningful component of $\vec{\gamma}$ is the one along $\vec{V}$. $\vec{\gamma}$ follows exactly the reorientation of $\vec{V}$ as $\mathbf{R}$ varies; this is the essence of the adiabatic approximation, to which the small $\hbar$ limit is found to be equivalent. If all components of $\vec{V}$ are vanishing so that the adiabatic approximation becomes meaningless, this classical limit just derived loses its signification, and one should resort to other limits and approximations, as shown in the next section. Equation of evolution of the longitudinal component (along $\vec{V}$ ), say $\gamma_{\zeta}$, in the adiabatic approximation, with $\hbar \rightarrow 0$ and in the spinorial formalism reads

$$
\begin{aligned}
& \frac{\partial \gamma_{0}}{\partial t}=\left\{H_{0}, \gamma_{0}\right\}+\left\{V_{\zeta}, \gamma_{\zeta}\right\}, \\
& \frac{\partial \gamma_{\zeta}}{\partial t}=\left\{H_{0}, \gamma_{\zeta}\right\}+\left\{V_{\zeta}, \gamma_{0}\right\} .
\end{aligned}
$$

This adiabatic limit is relevant as long as the Bohr frequency of the electron $\omega_{e}$ is much larger than the typical rate of change of the $\vec{V}$ vector [16]; expressed in energy scale, one must have

$$
\hbar \omega_{e} \gg \lambda_{N}\left|\frac{d V_{a}}{d \mathbf{R}}\right|
$$

where $\lambda_{N}=\hbar /|\mathbf{P}|$ is the wavelength of the nucleus and |l is any suitable norm. The change of the coupling, expressed in the energy scale of the electron, is much smaller than the de Broglie wavelength of the nuclei. This makes clear that the semiclassical limit joins here the adiabatic limit.

\section{B. One-center approximation}

In this section, we derive the classical limit in a different way: electronic degrees of freedom remain quantal while nuclear degrees of freedom become effectively classical. Let us write the Heisenberg equations from Hamiltonian (3) for the quantum operators [17] position $\hat{\mathbf{R}} \sigma_{0}$, momentum $\hat{\mathbf{P}} \sigma_{0}$ and the dimensioned spin $1 / 2$ matrices $\hat{S}_{a}=\frac{1}{2} \hbar \sigma_{a}$ :

$$
\begin{aligned}
\dot{\hat{\mathbf{R}}} \sigma_{0} & =\frac{\widehat{\mathbf{P}} \sigma_{0}}{M} \\
\dot{\hat{\mathbf{P}}} \sigma_{0} & =-\frac{\partial V_{0}}{\partial \widehat{\mathbf{R}}} \sigma_{0}-\frac{1}{\hbar} \sum_{a} \frac{\partial V_{a}}{\partial \widehat{\mathbf{R}}} \hat{S}_{a} \\
\dot{\hat{S}}_{a} & =\frac{1}{\hbar} \sum_{b, c} \epsilon_{a b c} \hat{V}_{b} \hat{S}_{c} .
\end{aligned}
$$

Using the Ehrenfest theorem to derive the classical behavior of a system consists in taking the averaged values of the operators on quantum states that are initially wave packets and are supposed to remain so, ideally, for an infinite time. We assume that in the energy region of interest, the density of states is sufficient to fulfill the condition on the wave packets states mentioned above: this is the classical object. Besides, the electronic Bohr frequencies $V_{a} / \hbar=\omega_{\mathrm{el}, \mathrm{a}}$ remain unchanged, being quantal quantities. A similar approach may be found in the literature $[9,10]$. In our case, the remaining quantum part of our Hamiltonian consists in finite dimensional operators, so that the evolution operator will consist only of ordinary differential equations, as will be seen. Ehrenfest equations are thus

$$
\begin{aligned}
& \frac{d\left\langle\widehat{\mathbf{R}} \sigma_{0}\right\rangle}{d t}=\left\langle\frac{\widehat{\mathbf{P}} \sigma_{0}}{M}\right\rangle \\
& \frac{d\left\langle\widehat{\mathbf{P}} \sigma_{0}\right\rangle}{d t}=-\left\langle\frac{\partial V_{0}}{\partial \widehat{\mathbf{R}}} \sigma_{0}\right\rangle-\left\langle\sum_{a} \frac{\partial \omega_{\mathrm{el}, a}}{\partial \widehat{\mathbf{R}}} \hat{S}_{a}\right\rangle \\
& \frac{d\left\langle\hat{S}_{a}\right\rangle}{d t}=\sum_{b, c} \epsilon_{a b c}\left\langle\omega_{\mathrm{el}, b} \hat{S}_{c}\right\rangle .
\end{aligned}
$$

Now, another difficulty appears: the Bohr frequencies $\vec{\omega}_{\text {el }}$ are nonuniform in space, that is, they depend on the operator $\widehat{\mathbf{R}}$ which is not a simple independant parameter as time could be. In order to use the intuitive notion of wave packets rather than the operators of the Heisenberg picture, let us return to the Schrödinger picture. The average values dealt with in Eqs. (16)-(18) are of the form

$$
\langle F(\widehat{\mathbf{R}}, t) \hat{\sigma}(t)\rangle \stackrel{\text { Schr.pict. }}{=}\left\langle c_{1}(\mathbf{R}, t), c_{2}(\mathbf{R}, t)|F(\widehat{\mathbf{R}}) \hat{\sigma}| c_{1}(\mathbf{R}, t), c_{2}(\mathbf{R}, t)\right\rangle,
$$

where $\hat{\sigma}$ represents any $2 \times 2$ operator. In this picture, it is easy to realize that, in general, it is not true that for all times the mean value of the product is equal to the product of the mean values

$$
\langle F(\widehat{\mathbf{R}}) \hat{\sigma}\rangle \stackrel{?}{=}\langle F(\widehat{\mathbf{R}})\rangle\langle\hat{\sigma}\rangle
$$

We are thus led to make the following crucial approximation: averaged values will be taken on particular 
quantum states $\left|\psi\left(\mathbf{R}_{\mathrm{cl}}\right)\right\rangle=\left|c_{1}\left(\mathbf{R}_{\mathrm{cl}}, \mathbf{t}\right) \mathbf{c}_{\mathbf{2}}\left(\mathbf{R}_{\mathrm{cl}}, \mathbf{t}\right)\right\rangle$, where $c_{1}\left(\mathbf{R}_{\mathrm{cl}}, \mathbf{t}\right)$ and $c_{2}\left(\mathbf{R}_{\mathrm{cl}}, \mathbf{t}\right)$ represent wave packets localized in position and momentum around the same classical point $\mathbf{R}_{\mathrm{cl}}, \mathbf{P}_{\mathrm{cl}}$. Consequently, we suppose quite naturally that at the classical limit, the wave packet upper and lower components do not split in space during their evolution in time. This approximation is completely distinct from the adiabatic approximation we were forced into in Sec. II A. Here we do not need any further approximation to proceed. We straightforwardly derive the equations

$$
\begin{aligned}
\frac{d \mathbf{R}_{\mathrm{cl}}}{d t} & =\frac{\mathbf{P}_{\mathrm{cl}}}{M}, \\
\frac{d \mathbf{P}_{\mathrm{cl}}}{d t} & =-\nabla_{\mathbf{R}_{\mathrm{cl}}} V_{\mathbf{0}}\left(\mathbf{R}_{\mathrm{cl}}\right)-\nabla_{\mathbf{R}_{\mathrm{cl}}} \vec{\omega}_{\mathrm{el}}\left(\mathbf{R}_{\mathrm{cl}}\right) \cdot \vec{S}, \\
\frac{d \vec{S}}{d t} & =\vec{\omega}_{\mathrm{el}}\left(\mathbf{R}_{\mathrm{cl}}\right) \times \vec{S},
\end{aligned}
$$

where

$$
\left.\vec{S}\right|_{a}=\left\langle S_{a}\right\rangle
$$

This result is known [18] and [12] but the important limitation of the one-center approximation is not always pointed out.

Equations (21)-(23) are classical in the way that they deal with c-numbers $\left(\mathbf{R}_{\mathrm{cl}}, \mathbf{P}_{\mathrm{cl}}, S_{a} \equiv<\hat{S}_{a}>\right)$ through ordinary differential equations. Moreover, (21)-(23) connect the classical evolution of a particle located at $\mathbf{R}_{\mathrm{cl}}$, $\mathbf{P}_{\mathrm{cl}}$ with the quantum exchange of probability between the two electronic surfaces. The correlation between both sets of variables is the main feature of these equations. Another interesting property, already noticed [18], is that they can be derived from a Hamiltonian. Putting $\mathbf{P}$ conjugate variables of $\mathbf{R}$ and $s$ conjugate of $\phi, S_{\mathbf{a}}$ becomes function of $s$ and $\phi$ :

$$
\begin{aligned}
& S_{x}=\sqrt{\left|S_{0}\right|^{2}-s^{2}} \cos \phi, \\
& S_{y}=\sqrt{\left|S_{0}\right|^{2}-s^{2}} \sin \phi, \\
& S_{z}=s
\end{aligned}
$$

the Hamiltonian reads:

$$
\mathcal{H}(\mathbf{R}, \mathbf{P}, \phi, s)=\frac{\mathbf{P}^{2}}{2 M}+V_{0}(\mathbf{R})+\vec{\omega}(\mathbf{R}) \cdot \vec{S}(\phi, s) .
$$

We derive such a result by noting that $(21)-(23)$ are in fact determined by the commutation relations of the operators $\hat{\mathbf{R}}, \hat{\mathbf{P}}$, and $\hat{S}_{a} ;$ the classical variables $\mathbf{R}, \mathbf{P}$, and $S_{a}$ share the same relations expressed in the Poisson brackets:

$$
\begin{aligned}
\{A(\mathbf{R}, \mathbf{P}, \phi, s), B(\mathbf{R}, \mathbf{P}, \phi, s)\}= & \frac{\partial A}{\partial \mathbf{R}} \frac{\partial B}{\partial \mathbf{P}}-\frac{\partial B}{\partial \mathbf{R}} \frac{\partial A}{\partial \mathbf{P}} \\
& +\frac{\partial A}{\partial \phi} \frac{\partial B}{\partial s}-\frac{\partial B}{\partial s} \frac{\partial A}{\partial \phi}
\end{aligned}
$$

Hence, a simple substitution in the Hamiltonian (3) of the operators by the previously mentioned variables is sufficient to derive its classical counterpart.
Returning now to the physical problem, it is the last term of Eq. (22) that couples the nuclear and the electronic degrees of freedom. It appears as coming from the $\vec{\omega}(\mathbf{R}) \cdot \vec{S}(\phi, s)$ term. It is then easy to realize that besides exceptional cases, this coupling makes the Hamiltonian nonintegrable. This is the first indication that chaos may arise when electronic and nuclear degrees of freedom are coupled together. The existence of a classical scalar Hamiltonian is then a particularly crucial property, as it allows one to use the whole standard machinery of classical mechanics to study chaos.

Earlier technique, known as "surface hopping", dealt with the case of surface crossing and led to the derivation of Poincaré surface of section. As ours, it is a hemiquantal treatment but in a different way. It consists of imposing that the particule remains in one diabatic surface at a time - hence its classical motion - the transitions between surfaces, occuring at the intersection, are randomly monitored according to the probability given by the Landau-Zener formula. With integrable diabatic surfaces, chaos has been pointed out [8]. It results from random transitions between the integrable tori of the two surfaces. The classical model developed here, allows to populate both surfaces which conforms better to the physical behavior. Moreover, as the coupling increases, the classical Hamiltonian (28) allows to exhibit breaking of tori, belonging to the whole spin-position phase space, as a signature of chaos.

\section{Liouville equations}

As this point, we make contact between the one-center approximation just described and the adiabatic limit obtained by the Wigner transform, in the spinorial formalism, Eqs. (8) and (9). Let us thus derive Liouville equations associated with the Hamiltonian (28). As stated above, the canonical variables for $\vec{S}$ are $s$ and $\phi$. The Liouville density function is thus $f=f(\mathbf{R}, \mathbf{P}, \phi, s)$. The equivalent quantities to $\gamma_{a}$ are

$$
\begin{aligned}
f_{a} & =\int f(\mathbf{R}, \mathbf{P}, \phi, s) S_{a} d s d \phi \\
f_{0} & =\int f(\mathbf{R}, \mathbf{P}, \phi, s) d s d \phi
\end{aligned}
$$

One could define similarly higher moments, such as $f_{x^{2}}, f_{x y}, \ldots$ The Liouville equations for $f_{0}, f_{a}$, and $a=x, y, z$ are

$$
\begin{aligned}
& \frac{\partial f_{0}}{\partial t}=\left\{H_{0}, f_{0}\right\}_{\mathbf{R}, \mathbf{P}}+\left\{\omega_{a}, f_{a}\right\}_{\mathbf{R}, \mathbf{P}} \\
& \frac{\partial f_{a}}{\partial t}=\left\{H_{0}, f_{a}\right\}_{\mathbf{R}, \mathbf{P}}+\left\{\omega_{b}, f_{b a}\right\}_{\mathbf{R}, \mathbf{P}}+\epsilon_{a b c} \omega_{b} f_{c} .
\end{aligned}
$$

In these equations, we assume summation over repeated indices; as indicated, the Poisson brackets imply derivation over $\mathbf{R}$ and $\mathbf{P}$ only.

In both equations, the left-hand side and the first Poisson bracket correspond together to a total time derivative in phase space (particle transport), the last term has an 
exact counterpart in the quantum dynamics. The second term of Eq. (32) is unexpected and harmful: it couples the momentum of order one $f_{a}$ to higher order momenta $f_{a b}$. A whole hierarchy of equations is thus created, as $f_{a b}$ itself follows a similar equation. It is this term that forbids to make the complete analogy between the Bloch equations and the equations derived by taking a classical limit of the spinorial Wigner distribution (6)-(7). To try to recover, let us pretend that we somehow cut this hierarchy, for example by explicitly imposing that moments of order higher than one are identically zero (a classical spin 1/2): $f_{a^{2}}=\frac{1}{3} S_{0}\left(S_{0}+1\right) f_{0}=\frac{1}{4} f_{0}$. The second term of Eq. (33) reduces then to the second term of Eq. (7). The Liouville equations seem now to represent the classical dynamics of a spin $1 / 2$. This may seem a sensible procedure because in the case of a heavy spinning particle in an inhomogeneous magnetic field these Liouville equations are the limit of the quantum equations because the cutoff of moments of order larger than $2 S_{0}$, which occurs in the quantum case and makes the difference between classical and quantum hierarchies of equations, occurs for higher and higher order when $S_{0} \rightarrow \infty$. However, by the same token, when applied to a finite value of $S_{0}$, and especially to $S_{0}=1 / 2$, we lost the usual interpretation of the Liouville equations for they cannot anymore be reduced to a set of ordinary differential equations, representing the evolution of a cloud of particles. All the simplicity of Hamiltonian dynamics is lost, and we cannot proceed further than Eq. (32).

As a conclusion to this section, we have shown that we are led into two types of approximation. (i) We strictly retain the idea of a spin $1 / 2$, and a proper classical limit. This formalism is sensible within the adiabatic transport formalism [19] but does not seem to say anything about mode conversion. (ii) On the other hand the hemiquantal approach, which we have developed here for a spin coupled to spatial degrees of freedom, allows for diabatic or adiabatic transitions. It is incompatible with the above-mentioned approximations, and supposes only one particle, characterized in configuration space as well as in spin space.

\section{SOME APPLICATIONS}

Early treatments of a mixed semiclassical-quantum system date back to Landau, Zener, and Stückelberg. Through different approaches, they derived the same well-known Landau-Zener formula. An important consequence concerning total cross sections of inelastic scattering was later obtained by Rosenthal. In this section, we shall compare these results obtained within quantum mechanics with our classical framework.

\section{A. Landau-Zener crossing}

Although the Bloch-like Eqs. (21)-(23) are obtained within the one-center approximation, we shall show that they reproduce some interesting effects that have not yet been examined in this purely classical framework. Before discussing in some details the Rosenthal-Stückelberg oscillations, let us devote some space for the description of a single Landau-Zener crossing. The Zener approach [1] is quite similar to ours, in the extent that the scattered particle is supposed to be a single wave packet. Without the coupling term $\nabla_{\mathbf{R}_{\mathrm{cl}}} \vec{\omega}_{\mathrm{el}}\left(\mathbf{R}_{\mathrm{cl}}\right) \cdot \vec{S}$ Eqs. (21)-(23) give the position $\mathbf{R}(t)$ of the particle, Eq. (21) being then equivalent to the Zener Hamiltonian. The importance and originality of the coupling term is that the motion is no longer independant of the wave amplitudes, as was the case in Zener as well as Stückelberg formulations. The asymptotic solution of the Landau-Zener equations are known in one single spatial dimension $R$, for a linear potential $V_{|1>,| 2>}(R)= \pm F_{z} R= \pm V_{z} / 2$ and for a constant coupling $V_{12}(R)=k=V_{x} / 2$. Assuming that the speed $v$ of the particle is constant, one has for the probability of transition (in our notations)

$$
P=\frac{1-S_{z}(+\infty) / S_{0}}{2}=e^{-2 \pi \Gamma},
$$

where the rate is

$$
\Gamma=\frac{1}{\hbar} \frac{k^{2}}{v F_{z}}=\frac{1}{2 \hbar} \frac{V_{x}^{2}}{d\left(V_{z}\right) / d t} .
$$

We remember that in classical terms the Larmor frequency around the longitudinal pseudomagnetic field is $V_{z} / \hbar=\omega_{\text {Larmor, } \|}$ and similarly for $V_{x} / \hbar=\omega_{\text {Larmor }, \perp}$. In the image of a classical angular momentum precessing around a pseudomagnetic field $\vec{V}$ (units of energy), the rate is now

$$
\Gamma=\frac{\omega_{\text {Larmor }, \perp}^{2}}{2 d \omega_{\text {Larmor }, \|} / d t}=\frac{\omega_{\text {Larmor }, \perp}^{2}}{2 v d \omega_{\text {Larmor }, \|} / d R} .
$$

These expression are purely classical, provided that we make the identifications pseudospin-angular momentum and pseudomagnetic field-true magnetic field. The diabatic potentials we chose are shown in Figure 2: the two sheets correspond to $V_{z}=v_{0} / 2 \tanh \left(F_{z} R / v_{0}\right)$. They cross at $R=0$, with difference slope $F_{z}$. The coupling term $V_{x}=k$ is damped with a Gaussian factor.

As a first example, let us show the influence of the field gradient term on the dynamics generated by the Hamiltonian (28): While the situation presented is as simple as possible, it serves as an illustration for more complicated effects that will appear for more complex potential sheets. First, let us recall the two opposite extreme situations: fast diabatic passage and slow, adiabatic passage through the crossing. Figure 3 shows in the diabatic frame of reference how the $\vec{\omega}_{\mathrm{el}}$ vector describes the crossing and how the $\vec{S}$ vector evolutes. Of course, $\vec{\omega}_{\text {el }} \cdot \vec{S}$ is conserved for adiabatic passage, in any frame of reference. In the diabatic passage (and in any frame) $\left.\vec{\omega}_{\mathrm{el}} \cdot \vec{S}\right|_{R=-\infty} \simeq-\left.\vec{\omega}_{\mathrm{el}} \cdot \vec{S}\right|_{R=+\infty}$.

In Fig. 4, we show the evolution of the populations of the two levels, as represented by the projection $S_{z} / S_{0}$ of the pseudospin on the $O_{z}$ axis, in the diabatic frame. This axis does change its orientation as one proceeds through the crossing at $R=0$. For a slow speed 


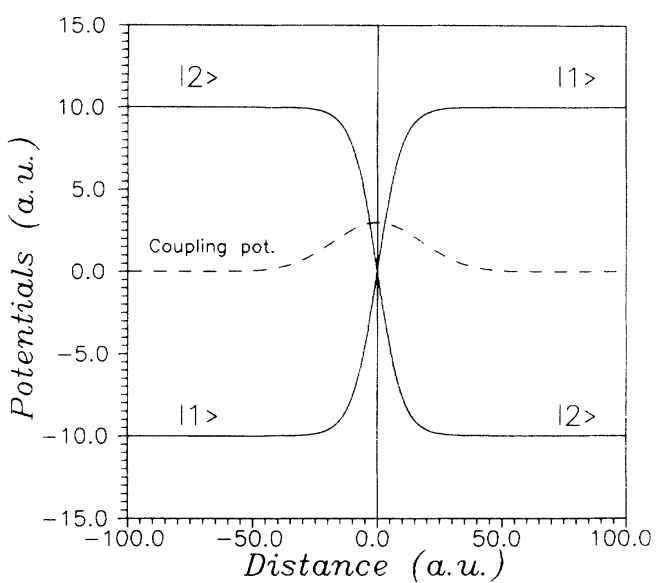

FIG. 2. The two potential lines used in the Landau-Zener type of crossing. All constants are in a.u. $\left(e=\hbar=m_{\text {electron }}=1\right)$. As in Fig. 1 , they cross each other at $R=0$. The off-diagonal potential term is damped by a Gaussian factor, characteristic length $r_{d}=5$; it is represented here by the dashed line. The diagonal potentials $V_{|1\rangle}, V_{|2\rangle}$ are depicted in full lines. Mass is taken at $M=1836.4$ (ratio of nuclear to electronic masses).

$\left[v(R=-\infty) \sim v_{0}=0.15\right.$ a.u., nearly adiabatic motion], $S_{z} / S_{0}$ nearly turns over, as expected. Furthermore, it undergoes oscillations around the $R=0$ position, as the axis of precession is nearly perpendicular to the $O_{z}$ axis. These oscillations are nearly identical with a restricted dynamics, if we artificially forget the coupling term. The

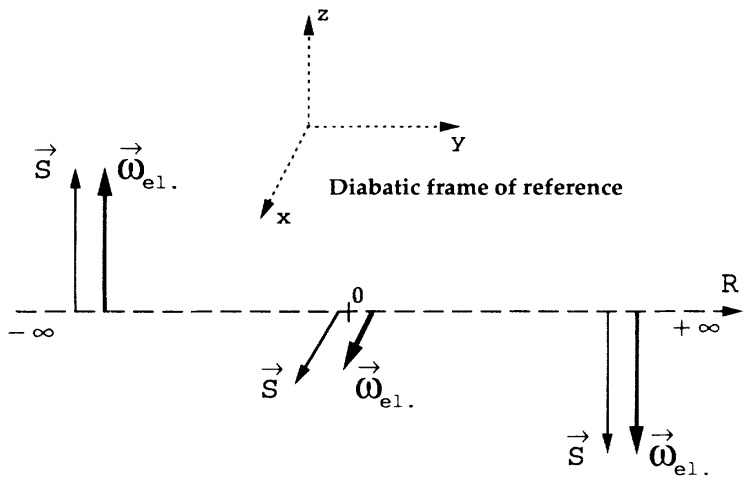

Adiabatic passage

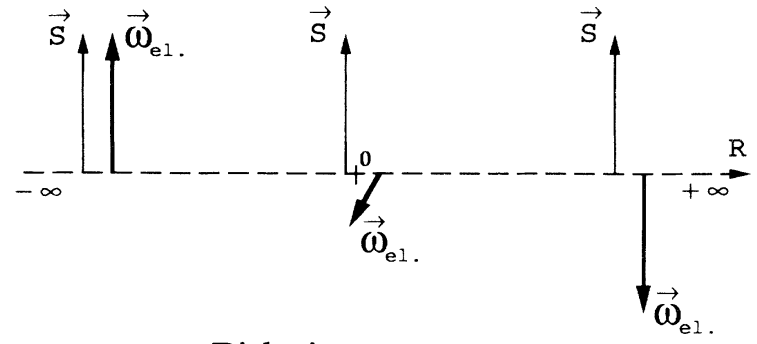

Diabatic passage

FIG. 3. Scheme of the adiabatic and diabatic passages through one Landau-Zener crossing, in the diabatic frame of reference.

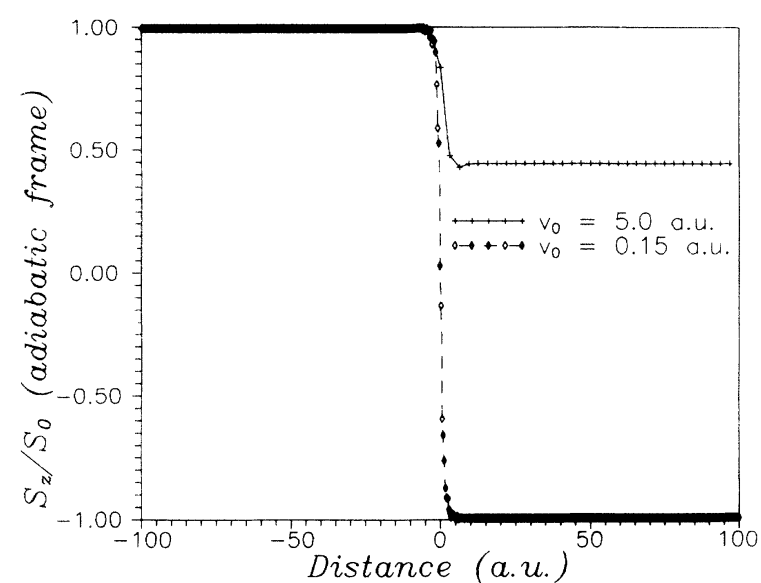

FIG. 4. Evolution of the normalized pseudospin component $S_{z} / S_{0}$, through the crossing, at a large speed (largely diabatic crossing) and moderate speed (more or less adiabatic crossing). As the pseudomagnetic field inverts from $R<0$ to $R>0$, the adiabatic transition (staying in the upper sheet) is represented by an change of sign of $S_{z} / S_{0}$ (diabatic frame representation). The oscillations nearby the crossing are due to the precession around the local $\vec{V}$ axis, distinct from the $O z$ direction.

opposite case, of a fast initial speed $v_{0}=5.0$ a.u. is also shown in the same figure.

Figure 5 shows the evolution of the speed through the crossing if we send a particle from $R=-\infty$ to $R=+\infty$ for a fast speed (initial speed $v_{0}=5.0$ ) or slow speed (initial speed $\left.v_{0}=0.15\right)$. As explained in the caption, both curves are normalized and display $v(R) / v(R=-\infty)$. In the adiabatic, slower motion, the particle loses much of its momentum when approaching the crossing but nearly regains it, as the gradient $\frac{\partial\left|\omega_{\mathrm{el}}\right|}{\partial R}$, and thus the force $\frac{\partial \omega_{\mathrm{el}} \cdot \mathbf{S}}{\partial R}$ changes sign. If $\Delta p$ is the overall change in momentum, one has in the adiabatic regime $\Delta p \simeq 0$. In the dia-

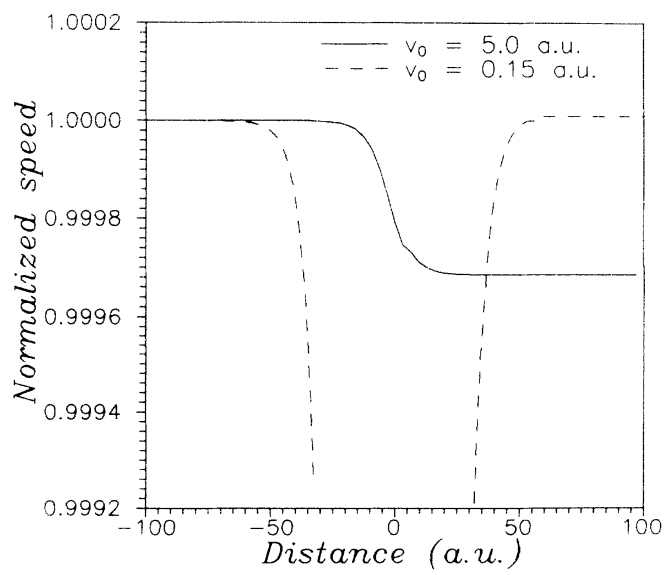

FIG. 5. Evolution of speed through the crossing at $R=0$. The two speeds are normalized in the figure as $\bar{v}=v / v_{0}$, so that each line seems to originate from the same asymptotic value. It it thus possible to show the relative variation in speed as the particle goes through the potential crossing. 


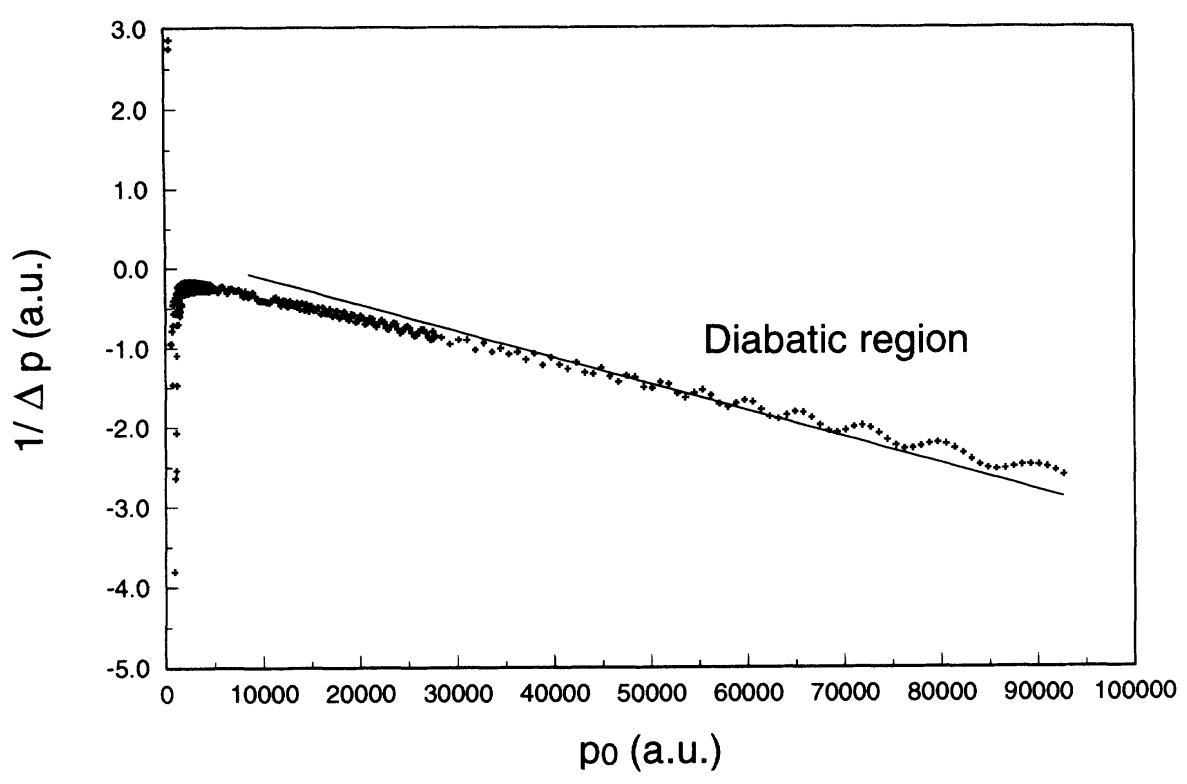

FIG. 6. Evolution of the inverse of the variation of the momentum $1 / \Delta p$ with respect to the incoming momentum $p$, for a single Landau-Zener crossing. Note the largely different scales of $p$ and $\Delta p$. The adiabatic regime is reached for very small momenta, $p \ll 1$. The diabatic regime shows the expected $1 / p$ behavior [Eq. (36)], with superimposed oscillations of small amplitude that are characteristic of the detailed dynamics.

batic regime, $\vec{S}$ changes sign through the crossing (diabatic frame of reference), so that the force $\frac{\partial \vec{\omega}_{e l} \cdot \vec{S}}{\partial R}<0$ always. $\Delta p$ is negative, and small in relative term with $p$, as $p^{2} / 2 M \gg \vec{\omega}_{\mathrm{el}} \cdot \vec{S}$, since $m / M \ll 1$. For $p$ sufficiently large, one has in the potentials of Fig. 2,

$$
\Delta p \simeq-\frac{\left(V_{z}(+\infty)-V_{z}(-\infty)\right) M}{p} .
$$

This behavior may be seen in Fig. 6. The detailed dynamics appear at large $p$ as oscillations superimposed to the general trend of $\Delta p$.

As a last example let us depict the Landau-Zener probability of transition, Fig. 7 , where we plot $P=P(1 / v)$, Eqs. (33)-(34). In the so-called restricted dynamics, we artificially turn off the coupling term (not a Hamiltonian motion anymore) while the term is kept in the full dynamics. Its overall effect is rather small, and tends to decrease the probability of transition at low speeds, as the particle tends to pass even slower over the transtion point, thereby increasing the adiabaticity.

\section{B. Rosenthal-Stückelberg oscillations}

In order to explain the oscillatory behavior of the total cross section of some molecule-atom inelastic cross section [7], Rosenthal found that the two output channels are coherently populated through a third channel, crossing the former ones at $R=R_{i}$ (see Fig. 8). From this point to the outer crossing at $R=R_{e}$, the two waves accumulate a phase difference $\delta$. The system makes then a single Landau-Zener passage through the outer crossing as previously. However in this case, the amplitudes are different from zero on both levels and in particular have this phase difference of $\delta$. The transition through the crossing acts as a measurement of $\delta$ because the population difference between the two channels depends on it. It has been shown the final population difference exhibits an oscillatory behavior as $\sin (\delta+\varphi)$, where $\varphi$ is a constant phase. Let us quickly derive the Rosenthal approximate expression for $\delta$. Let $V_{1}(R)$ and $V_{2}(R)$ be the adiabatic potentials 1 and 2 and $E$ the energy of the wave. The semiclassical spatial phase is

$$
\phi_{1,2}=\frac{1}{\hbar} \int_{R_{i}}^{R_{e}} \sqrt{2 m\left(E-V_{1,2}\right)} d R
$$

then $\delta=\phi_{1}-\phi_{2}$. In the limit $E \gg V_{1,2}$, we approach $\delta$ by

$$
\delta \simeq \frac{1}{\hbar} \sqrt{\frac{m}{2 E}} \int_{R_{i}}^{R_{e}}\left(V_{1}-V_{2}\right) d R
$$

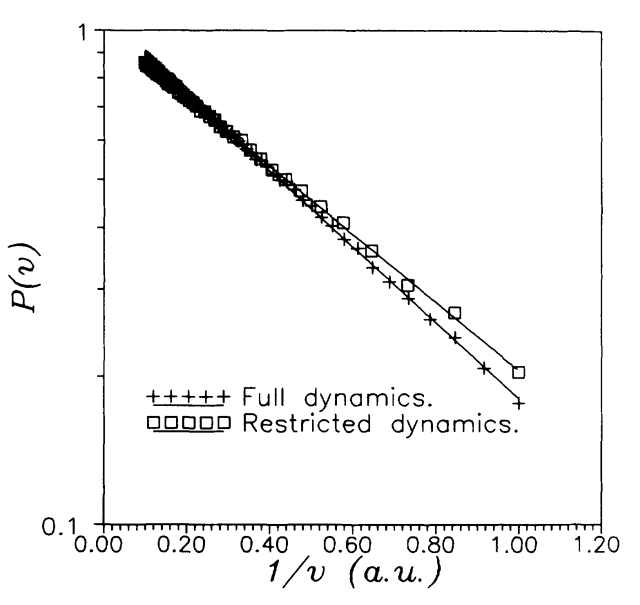

FIG. 7. Probability of diabatic transition $P(v)$, in logarithmic scale, as a function of the inverse of the incoming speed $1 / v$. The open squares result from a simulation where the coupling term is kept at zero; the equations of motion are identical to the original Zener ones, and the line describe Eqs. (33) and (34). The crosses result from a simulation with the coupling term included, thereby allowing for a change in speed during the crossing. The probability is slightly decreased at low speeds, as explained in the text. 


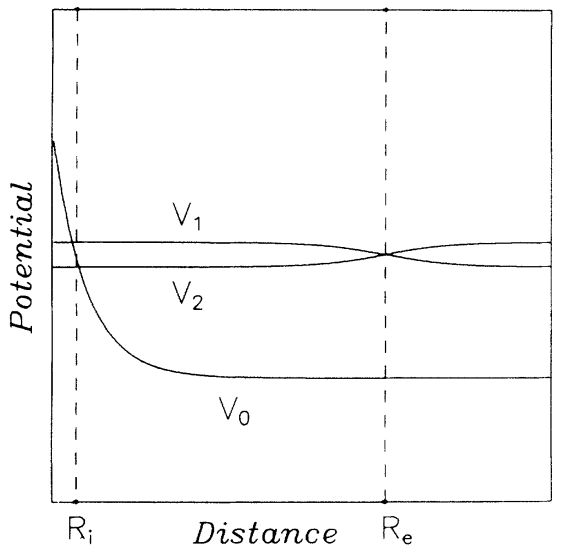

FIG. 8. Scheme of the level system resulting in Rosenthal oscillations of the total cross section, in the diabatic representation. The incoming channel 0 coherently populates the two outgoing channels 1 and 2 at $R=R_{i}$. These levels are coupled together by $\vec{V}=\left(V_{x}, 0, V_{z}\right)$, till the crossing at $R=R_{e}$, where $\vec{V}$ vanishes. For $R>R_{e}$, only $V_{z}$ is different from zero.

If $V_{1}(R=+\infty)=V_{2}(R=+\infty)=0$, then $\sqrt{\frac{m}{2 E}}=$ $v_{\infty}^{-1}$, the asymptotic speed. Hence we get the well-known Rosenthal formula,

$$
\delta \simeq \frac{1}{\hbar v_{\infty}} \int_{R_{i}}^{R_{e}}\left(V_{1}-V_{2}\right) d R .
$$

The proportionality of $\delta$ to the inverse speed leads to the sinusoidal behavior of the population difference as a function of $\frac{1}{v_{\infty}}$. These quantum quantities, i.e., difference of population and phase can be mapped onto classical quantities related to the angular momentum $\vec{S}$ defined in Sec. II. Writing the spinor $\left|c_{1} c_{2}\right\rangle$ as

$$
\left|\begin{array}{l}
c_{1} \\
c_{2}
\end{array}\right\rangle=\left|\begin{array}{l}
\left|c_{1}\right| e^{i \phi_{1}(R)} e^{-i E t / \hbar} \\
\left|c_{2}\right| e^{i \phi_{2}(R)} e^{-i E t / \hbar}
\end{array}\right\rangle
$$

one gets, through formalism of paragraph II B

$$
\begin{aligned}
S_{x}=\left\langle\hat{S}_{x}\right\rangle & =\frac{\hbar}{2} A\left(R_{c l}\right) \cos \left[\left(\phi_{1}-\phi_{2}\right)\left(R_{c l}\right)\right], \\
S_{y}=\left\langle\hat{S}_{y}\right\rangle & =\frac{\hbar}{2} A\left(R_{c l}\right) \sin \left[\left(\phi_{1}-\phi_{2}\right)\left(R_{c l}\right)\right], \\
S_{z}=\left\langle\hat{S}_{z}\right\rangle & =\frac{\hbar}{2}\left[\int\left(\left|c_{1}\right|^{2}-\left|c_{2}\right|^{2}\right) d R\right] .
\end{aligned}
$$

It is obtained under the assumption of a Gaussian wave packet centered at $R_{c l}$ and by making a development of $\phi_{1}-\phi_{2}$ around this point; $A\left(R_{c l}\right)$ is an amplitude depending on the particular form of the wave.

The phase difference $\delta=\phi_{1}-\phi_{2}$ is in our classical picture simply the accumulated angle of precession of $\vec{S}$ around the $O_{z}$ axis. This proves that it is possible to observe the Rosenthal quantal oscillations in the purely classical scheme, using Hamiltonian (28).

As an application, we numerically integrated equations of motion (21)-(23), for a situation similar to the experiments described in [20], see Fig. 8. The two asymptotic

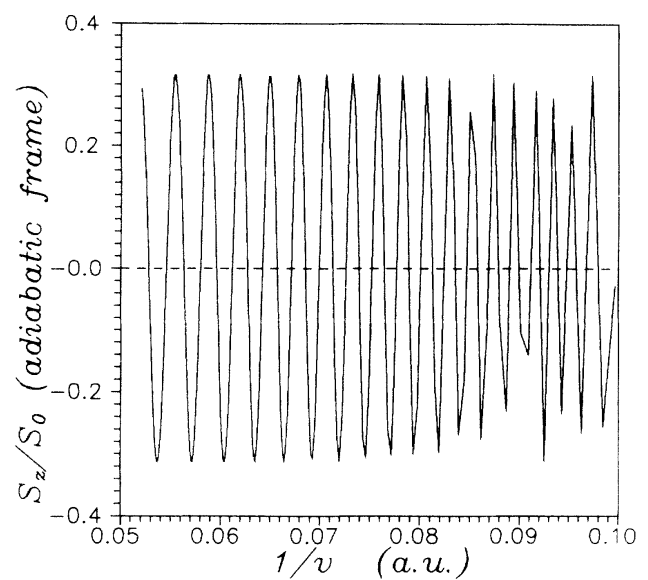

FIG. 9. Rosenthal oscillations in the polarization, after going through the outer crossing of Fig. 8. Purely classical simulation, with the coupling term included. The situation is formally similar to [20].

outgoing channels correspond to the $4{ }^{1} D\left(m_{L}= \pm 2\right)$ and $4^{1} F\left(m_{L}= \pm 2\right)$ levels of helium, that are coupled by the Stark field of the ion that excited it. These two coupled levels are separated by a small asymptotic energy of $V_{1}-V_{2}=10 \mathrm{~cm}^{-1} \simeq 10^{-4}$ a.u.. In the language of Fig. 8 and in a.u., one has $V_{x}=-q / R, V_{z}=10^{-4}, R_{i}=1$, and $R_{e}=650$. We send off a particle at $R_{i}$ for varying speeds $v$, in a pure $S_{z} / S_{0}=+1$ state in the laboratory frame $-S_{z} / S_{0} \simeq 0$ in the adiabatic frame. We keep track of the resulting total polarization $S_{z} / S_{0}$ in the asymptotic region, where $V_{x} \ll V_{z}$. A plot of $S_{z} / S_{0}=f(1 / v)$ is presented on Fig. 9. This polarization is exactly analogous, for a one-dimensional scattering "experiment", to the total cross section whose oscillations are characteristic of the Rosenthal-Stückelberg effect. Figure 9 shows these oscillations extremely clearly; the oscillatory regime extends for most initial speeds ( $v>1.1$, approximately). A Fourier analysis shows a predominance of the order of $10^{2}$ in the amplitude of the fundamental frequencies over any harmonic.

\section{Diffusive chaos}

As a last and most promising example, we shall describe in this section how the interaction of the spatial and angular degrees of freedom may induce classical chaos, in a 1D configuration space. This has already been noted in a Bloch model [13], and in general surface crossings $[3,8]$ but we shall concentrate in a sector that may bear importance for chemical reactivity, namely classical diffusive chaos [21-23]. The model chosen is as simple as possible and the analysis is just qualitative, as the detailed characterization of that chaos will be described elsewhere. Let us recall here that diffusive chaos may be characterized by the occurrence of very many singularities in classical deflection functions or cross sections. The singularities lie on a Cantor set [24] which is the image of the Cantor set of unstable periodic or quasiperiodic 


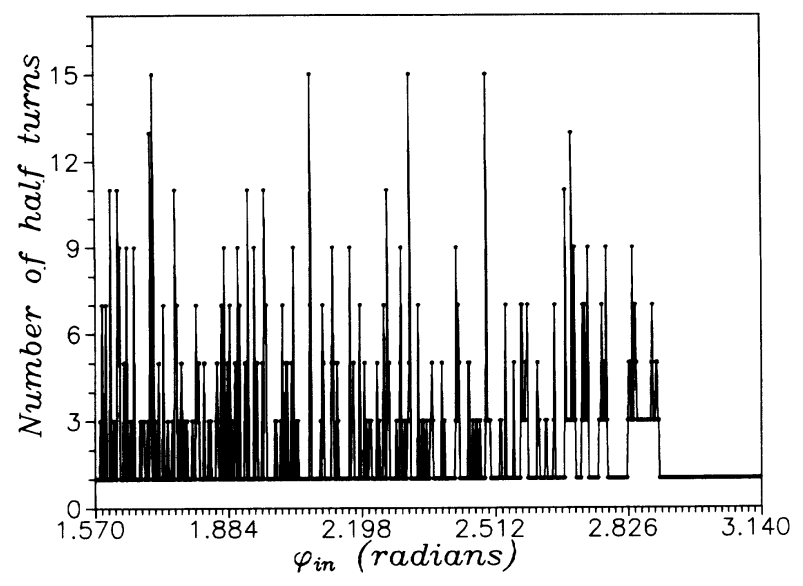

FIG. 10. Scattering experiment in the potentials of Fig. 1. The particle is sent in with a total energy of approximately -0.05 a.u. (see text). The overall deflection function $N=N(\phi)$ is shown with $\pi / 2 \leq \phi \leq \pi . N$ is the number of half turns of the trajectory; $N=1$ means direct scattering off the center. $\cos \phi=S_{z} / S_{0}$. The simulation is made of 1000 points, the solid lines serving as guide to the eyes.

orbits that are trapped in the interaction region. These orbits result from the interaction of two oscillators, one of them at least being nonlinear. If the coupling is strong enough, the whole series of resonances become unstable, and each member of the series interacts with each other to create unstable bound orbits, at positive energies. As the bound orbits are unstable, each nearby trajectory eventually reaches incoming and outgoing asymptotic regions. As a result, a number of scattering trajectories get trapped inside the interaction region, for an amount of time longer than the simple back and forth scattering. A signature of scattering chaos may thus be found in the function "Number of trapped orbits vs impact parameter" $N=N(b)$. The number and organization of the singularities of $N(b)$ is a faithful signature of the unstable periodic orbits that lie in the interaction region. For a chaotic scattering situation, the singularity pattern of $N(b)$ must show a self-similar structure, that is, singularities at all scales in $b$, for $N \rightarrow \infty$ each time the trajectory hits the vicinity of a quasiperiodic orbit.

We shall make here a one-dimensional scattering numerical experiment: trajectories start and end at infinity and oscillate a certain number of times nearby the center of diffusion at $r=0$. The impact parameter will be a given internal state $S_{z} / S_{0}$ that will be varied from trajectory to trajectory. At the end of the scattering event, one may recall the number of times the trajectory made halfturns: this constitutes the most simple signature of temporarily trapped orbits [25].

We present now the two diabatic potentials that enter into the formalism of Sec. IIB, as well as the potential coupling between them. We have of course two potential sheets $V_{\mathrm{I}}, V_{\mathrm{II}}$ (see Fig. 1). The nonlinear oscillator we have here is of Morse type (level I); level II is flat for all purposes, but is exponentially growing at very small distances, in order to prevent unphysical motion into $r \leq 0$. The coupling between the two levels $V_{z}=V_{\mathrm{I}}-V_{\mathrm{II}}$ is taken as a constant $V_{x}$. A smooth damping of that coupling would not change any of our findings, but would introduce unnecessary nonlinearities and parameters. This nonlinear Morse oscillator $V_{z}$ is coupled to the spin precession through the last term of Eq. (22), where $\vec{\omega}$ has to be understood as $\vec{V} / S_{0}=2 \vec{V}$ in atomic units $\left(\hbar=e=m_{e}=1\right)$. The particle will be sent off from infinity (asymptotic plane $r \rightarrow \infty, t=-\infty$ ), with some initial momentum $P$, spin projection $S_{z} / S_{0}$, and a phase $\phi=\arctan \left(S_{y} / S_{x}\right)$. This phase is ill defined in the asymptotic plane; one has in fact to resort to the phase the precessing spin would have at a given finite distance, if $V_{z}$ were constant. We take this $\phi=0$ always. In order to get some chaos, that is, some trapped trajectories, the $V_{\mathrm{I}}<0$ part must be reached for some values of $S_{z} / S_{0}$. Inside the domain $-1 \leq S_{z} / S_{0} \leq 1$, the part that may

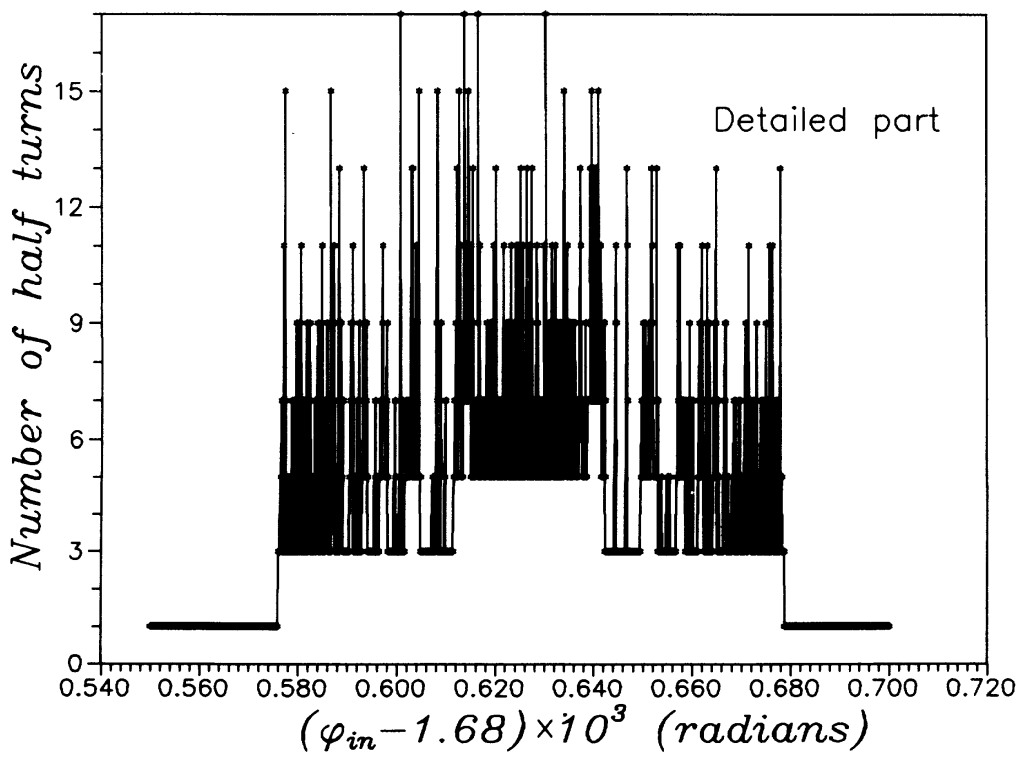

FIG. 11. A detail of the preceding figure, over 1500 points. Enlargment of the $\phi$ axis by a factor $10^{3}$, subtracting a constant value of $1.68 \mathrm{rad}$. The persistance of repetitive structures is obvious. 


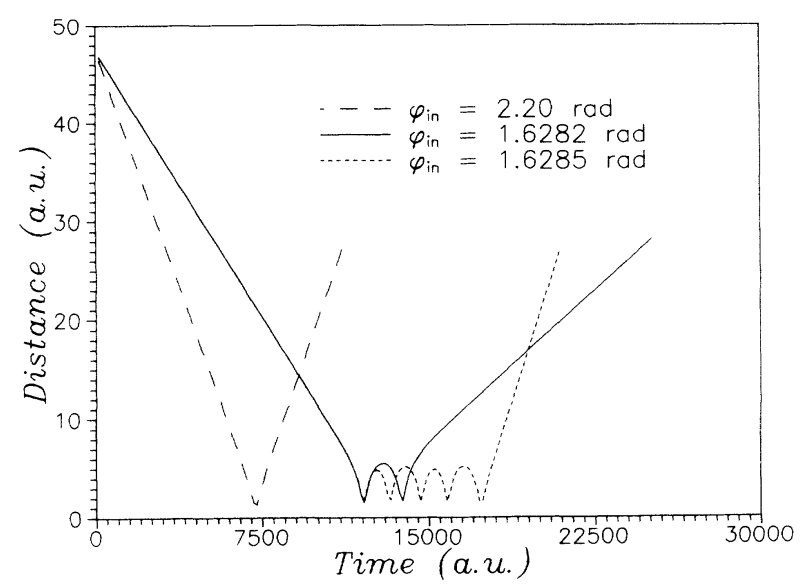

FIG. 12. Some trajectories in the $(r, t)$ plane, showing $1,3,9$ half turns. The quoted $\phi$ defines the initial $S_{z} / S_{0}=\cos \phi$ value. A high sensitivity to initial conditions is clear, either in this figure or in Fig. 11.

reach trapped orbits is smaller the larger the total energy $E$ is. We chose to work at $E=\left(V_{\mathrm{I}}+V_{\mathrm{II}}\right) / 2$. The kinetic energy $T$ increases from $T=0, S_{z}=S_{z}(\max )$ to $T=V_{I I}$ at $S_{z} / S_{0}=-1$. For the sake of convenience, we shall constantly label $S_{z} / S_{0}=\cos \phi$ by this angle $0<\phi \leq \pi$.

As a first result, we show the deflection function $N=$ $N(\phi)$ as a whole in Fig. 10, and a detailed part (approximate enlargment factor $10^{5}$ ) in Fig. 11. Both characteristics of chaotic scattering are clear: a great number of singularities and structures that persist at all levels. Simulation with subsequent enlargments would show a similar structure of the $N=N(\phi)$ function. To understand what is exactly happening, let us show some specific trajectories in the $(t, r)$ and $\left(r, S_{z} / S_{0}\right)$ planes, Figs. 12, 13. For $\phi_{\text {in }}=2.20$ - high kinetic energy - the particle is just reflected back, while around $\phi_{\text {in }}=1.68$, several complicated things occur. We see that two nearby trajectories diverge after collision and that they can be

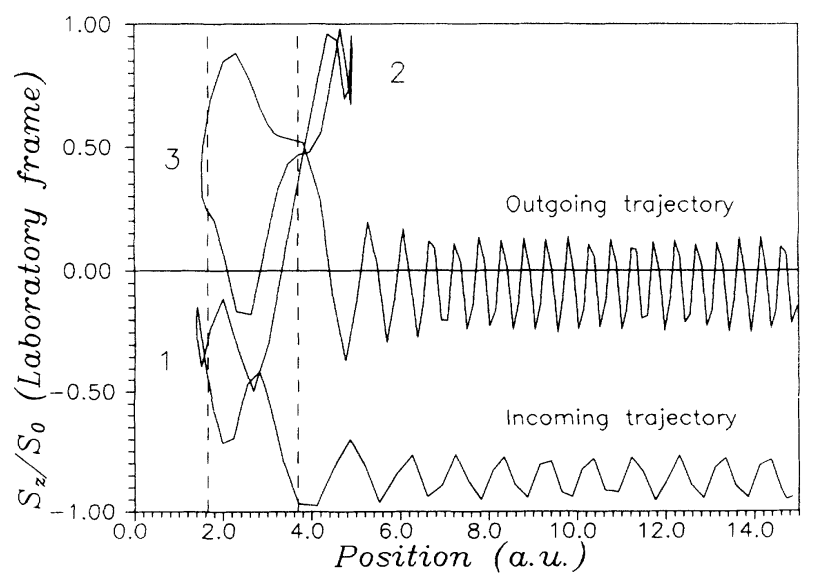

FIG. 13. The trajectory with $\phi=1.6282$ of Fig. 12, in the $\left(r, S_{z} / S_{0}=\cos \phi\right)$ plane. The dashed lines show $R_{i}$ and $R_{e}$ (Fig. 1). The strange appearance of the oscillations for $R \geq 6$ are due to the large step integration routines (Bülirsch-Stoer algorithms) that compute only a few points in the sinusoid, for a precision of about $10^{-8}$. trapped for several oscillations, actually for an arbitrary number of oscillations. Let us describe one such event in detail (Fig. 13). The particle comes in the lower half of the figure, with low $S_{z} / S_{0}$. In the first half turn (marked 1 ), the phase is such that the polarization changes towards +1 , in the lab frame (nearly fully towards $V_{\mathrm{I}}$ ). It is thus reflected back while climbing the hill (half turn 2). Polarization is destroyed after the half turn 3 , so that a nearly unpolarized particle goes away, with a small kinetic energy. It is clear that a very slight change in the incoming trajectory might result in a different kind of half turn 3 for example, allowing for one more trapped orbit. One understands also easily why self-similar structures appear, as the whole story repeats itself for each half turn, but each time on a finer scale for the incoming primary trajectory. A full analysis of this type of chaos is now underway, as well as some calculations on the corresponding quantum system.

\section{CONCLUSION}

We have analyzed in this paper two images of a heavy particle moving semiclassically in a two-sheet potential. A first image, that is known as adiabatic, forbids any mode conversion between the two adiabatic potential sheets. As an advantage, its derivation is rigorous within the Wigner transform formalism. It can be readily extended to any number of sheets. A second approach seem to be more adapted to deal with mode conversion (Landau-Zener crossings, conical points, and nonadiabatic transitions). If one imposes that wave packets remain localized around the same point in any potential sheet, then the Ehrenfest theorem has led us to a simple hemi-quantal formalism. We find a classical Hamiltonian, and the dynamics that it generates is easy to simulate. In passing, we have noted that the two approaches are mutually incompatible.

This simple classical model is sufficient to describe the Rosenthal oscillations that have been widely observed in atomic and molecular collision experiments. We also showed that the dynamics around a single crossing is similar to the Landau-Zener equations, except for a new term, that couples polarization to spatial motion. This term has its counterpart in pure quantum mechanics. It has little influence on a single Landau-Zener transition, but considerable effect if one of the levels supports bound states. Change of polarization during a crossing may temporarily trap a particle and even, as shown, lead to transient chaos. It is clear that the one-center classical image cannot cope with some simple quantum effects such as the Stern-Gerlach experiment, that the adiabatic image accomodates readily. The best blend of the two classical dynamics that could mimic a quantum evolution in several spatial dimensions on a many-sheets potential is still an open problem. This would be a formalization of the particle trajectory in an inelastic collision.

\section{ACKNOWLEDGMENTS}

The authors wish to thank M. Barrat (Lab. des Collisions Atomiques et Moléculaires, Orsay) for decisive encouragement. 
[1] C. Zener, Proc. R. Soc. London, Ser. A 137, 696 (1932).

[2] W.R. Thorson, J.B. Delos, and S.A. Boorstein, Phys. Rev. A 4, 1052 (1971); A. Joye and C.E. Pfister, Phys. Lett. A 169, 62 (1992); C. Zhu and H. Nakamura, J. Chem. Phys. 98, 6208 (1993).

[3] R.G. Littlejohn and W.G. Flynn, Chaos 2, 149 (1992).

[4] L. Landau and E. Lifshitz, Mécanique Quantique (Mir, Moscow, 1967), Sec. 53.

[5] T.C. Thompson, D.G. Truhlar, and C. Alden Mead, J. Chem. Phys. 82, 2392 (1985).

[6] C. Alden-Mead and D.G. Truhlar, J. Chem. Phys. 70, 2284 (1979); D. Dehareng, X. Chapuisat, J.C. Lorquet, C. Galloy, and G. Raseev, ibid. 78, 1246 (1983).

[7] H. Rosenthal, Phys. Rev. A 4, 1030 (1971).

[8] E.J. Heller, J. Chem. Phys. 92, 1718 (1990).

[9] D.J. Diestler, J. Chem. Phys. 78, 2240 (1983).

[10] D.V. Shalashilin, A.V. Michtchenko, and F. Lara, Chem. Phys. Lett. 207, 250 (1993).

[11] A. Delon, R. Jost, and M. Lombardi, J. Chem. Phys. 95, 5701 (1991).

[12] A. Bulgac and D. Kuznetsov, Nucl. Phys. A 545, 549c (1992).

[13] D. Hennig and B. Esser, Phys. Rev. A 46, 4569 (1992).

[14] M.S. Child, Semiclassical Mechanics with Molecular Ap- plications (Clarendon, Oxford, 1991).

[15] E.J. Heller, J. Chem. Phys. 65, 1290 (1976).

[16] A. Messiah, Mécanique Quantique (Dunod, Paris, 1964), Sec. XVII-13.

[17] Here, we properly make use of a distinct notation for quantum operators, such as $\hat{\mathbf{R}}$, and classical dynamical quantities, such as $R ; \sigma_{0}$ is the identity $2 \times 2$ matrix.

[18] H.D. Meyer and W.H. Miller, J. Chem. Phys. 71, 2156 (1979).

[19] R.G. Littlejohn and W.G. Flynn, Phys. Rev. A 45, 7697 (1992); R.G. Littlejohn and S. Weigert, ibid. 48, 924 (1993).

[20] M. Carré, A. Zgainski, M. Gaillard, M. Nouh, and M. Lombardi, J. Phys. (Paris) 42, 235 (1981).

[21] B. Eckhardt, Physica D 33, 89 (1988); P. Gaspard and S.A. Rice, J. Chem. Phys. 90, 2225 (1989); S. Bleher, C. Grebogi, and E. Ott, Physica D 46, 87 (1990); L. Bonnet, J.C. Rayez, and Ph. Halvick, J. Chem. Phys. 99, 1771 (1993).

[22] L. Wiesenfeld, J. Phys. B 25, 4373 (1992).

[23] C. Jung, J. Phys. A 19, 1345 (1986).

[24] Loosely speaking, an overcountable set of singularities, but still of Lebesgue measure zero.

[25] Z. Kovács and L. Wiesenfeld (unpublished). 